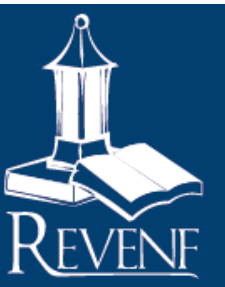

\title{
Pertinencia del plan de estudios de la Licenciatura en Enfermería de la Universidad de Costa Rica con el contexto socio-laboral ${ }^{1}$
}

Institución: Universidad de Costa Rica

\section{COMO CITAR}

Rojas, L.. (abril, 2013). Pertinencia del plan de estudios de la Licenciatura en Enfermería de la Universidad de Costa Rica con el contexto sociolaboral. Rev. Actual en Costa Rica, 24, 1-16. Recuperado de:: <http://www.revenf.ucr.ac.cr/pertinencia.pdf>ISSN 1409-4568

\section{RESUMEN}

El objetivo de este artículo es presentar los resultados de una investigación educativa, la cual analizó la pertinencia del plan de estudios en el contexto socio-laboral de la carrera de Licenciatura en Enfermería de la Universidad de Costa Rica. La población estuvo constituida por las y los egresados de instituciones públicas y privadas de la generación 2008 y por los entes empleadores en donde laboraban los egresados. Se aplicó un muestreo probabilístico simple; para la muestra de entes empleadores se eligió 6 instituciones en donde trabajaban los individuos seleccionados en la muestra. Se analizaron tres dimensiones: la formación del egresado, el desempeño en campos no tradicionales y la trascendencia de la acción laboral en la atención a la persona. Para la recolección de los datos, se aplicó un cuestionario estructurado y una entrevista semiestructurada a informantes claves. El estudio determinó que la propuesta educativa correspondiente a la carrera de Licenciatura en Enfermería de la Universidad de Costa Rica se encuentra en relación con el contexto socio-laboral. No obstante, los procesos educativos deben estar en continua revisión, actualización y mejoramiento, de acuerdo con los planteamientos que surgen a través del tiempo, y con el objetivo de que el estudiantado se asegure que su formación responderá a las exigencias sociales, laborales y en salud. Se concluye, entonces, que existe coherencia entre la formación de los y las egresadas y sus campos de desempeño laboral. No obstante, se requiere de mayor tiempo para el desarrollo de experiencias de aprendizaje en los módulos y en el curso de Gestión del Cuidado, así como de mejoras internas en relación con la suficiencia e idoneidad de tales experiencias; esto con la finalidad de alcanzar el constante mejoramiento de la oferta educativa.

Palabras clave: Contexto-socio-laboral, Curriculum, Enfermería

\footnotetext{
${ }^{1}$ Fecha de recepción: 22 de enero del 2013

Fecha de aceptación: 12 de febrero del 2013

${ }^{2}$ Profesora catedrática de la Universidad de Costa Rica. Enfermera Obstetra. Doctora en Educación y Magister en Evaluación Educativa. Correo electrónico: ligiarojas7@gmail.com
} 


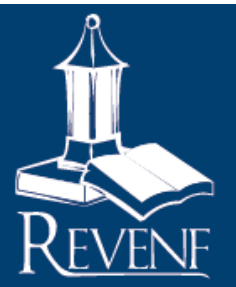

\title{
Pertinence of the curriculum of the Degree of Nursing at the University of Costa Rica with the socio-labor context ${ }^{1}$
}

Institution: University of Costa Rica

Ligia Rojas Valenciano $^{2}$

CITED

Rojas, L.. (abril, 2013). Pertinence of the curriculum of the Degree of Nursing at the University of Costa Rica with the socio-labor context. Rev. Actual en Costa Rica, 24, 1-16. Recovered:: <http://www.revenf.ucr.ac.cr/pertinencia.pdf> ISSN 1409-4568

\begin{abstract}
The objective of this paper is to present the results of educational research which analyzed the pertinence of the curriculum in the context of socio-labor Bachelor in Nursing at the University of Costa Rica. The population consisted of the graduates and public and private institutions of generating 2008 and the employing bodies where graduates worked. We applied a simple probabilistic sample was chosen employing bodies where six institutions were working the graduates and selected in the sample. We analyzed three dimensions: graduate training, performance in nontraditional fields and significance of industrial action in the person's attention. To collect data, a questionnaire was structured and semi-structured interviews with key informants. The study found that the educational proposal for the Bachelor in Nursing at the University of Costa Rica, is closely pertinence to the socio-labor. However, the educational process must be constantly revised, updated and improved according to the proposals that emerge over time for the students to ensure that your training will meet the requirements in health, social and employment. We conclude that there is a strong coherence between training and job performance fields and graduated from. However, more time is required for the development of learning experiences in the course modules and care management, and improve internal to everyone adequacy and appropriateness of such experiences, in order to be in constant improvement of educational provision.
\end{abstract}

Keywords: Context-socio-labor, Curriculum, Nursing

\footnotetext{
${ }^{1}$ Date of receipt: 22 de enero del 2013

Date of acceptance: 12 de febrero del 2013

2 Professor of the University of Costa Rica. Obstetric nurse. Doctor of Education and Master of Educational Evaluation.

Email: 1igiarojas7@gmail.com
} 


\section{INTRODUCCIÓN}

En general, los docentes universitarios tienen experticia en la profesión en la cual se desempeñan; es escaso el profesorado de una unidad académica universitaria que maneja una plena noción de la elaboración, confección y relevancia de los planes de estudio. El hecho más notable es el de haber recibido una instrucción corta sobre la didáctica universitaria de la que se valen para desempeñarse como docentes. De igual forma, son escasos los interesados en incursionar en la docencia universitaria como carrera adicional a su profesión base. Por esta razón, algunos planes de estudio muestran mayores aciertos que otros, lo cual significa que el estudiantado recibe una formación poco pertinente desde los diferentes aspectos que conforman el plan de estudios.

Primero que todo, es importante definir qué es un plan de estudios y cuáles son los elementos que confluyen en él. Este puede definirse como “...sinónimo de currículo, que a su vez deriva del vocablo latín curriculum que significa pista de carreras. Es decir la trayectoria que un corredor o un caballo debe seguir para concluir una carrera." (Departamento desarrollo sostenible de la FAO, s.f. p.1)

Según Rojas (2006), el proceso educativo está compuesto por diferentes fenómenos y características; una de ellas es la intencionalidad, la cual es imprescindible para añadir una cuota de desarrollo, crecimiento y maduración en el ser humano. Estas intenciones responden a intereses, motivos, aspiraciones y expectativas tanto del estudiantado como del profesorado. Otra característica importante es la emanación de una necesidad cultural y de una función social (p.24). Esta última se desarrolla por la influencia de la sociedad sobre la instituciones educativas -en este caso particular- la universidad.

De acuerdo con Brenes y Porras, (1995) se dice que: "Este es el motivo por el cual se ha descargado en la institución escolar la función social de perpetuar la cultura a través de las generaciones y los siglos" (p.226). Por consiguiente, la educación se convierte en un pilar de trascendental importancia para el desarrollo de la sociedad y para la transmisión de la cultura, porque las generaciones se alimentan unas de otras y, así, se prepara al ser humano para adaptarse al medio que le rodea.

Otra característica interesante del proceso educativo es su vinculación con el de desarrollo y crecimiento físico, mental y psicológico del ser humano. Así, según Brenes y Porras (1995), "es un proceso para todas las personas desde sus propias capacidades de desarrollo intelectual; de ahí que se considere como un proceso complejo" (p.227)

El fenómeno educativo está influido por aspectos sociales, históricos, ideológicos y políticos, los cuales condicionan una determinada realidad educativa. Todos estos aspectos afectan, positiva o negativamente, el proceso educativo tanto de manera directa como indirecta. De esta forma, Brenes y Porras (1995) mencionan que, "alrededor del fenómeno educativo, existen factores externos al sistema educativo, hechos, procesos, y diversos factores. Se pueden mencionar el medio, la estructura etnocaracteriológica y socioeconómica, el desarrollo cultural, científico y tecnológico y las corrientes educativas, entre otros aspectos.”(p.230)

Retomando los párrafos iniciales, los estudiosos del curriculum mencionan que existe una gran cantidad de posturas generadas por una honda preocupación y el interés concerniente a la teoría y la práctica educativa. Al respecto, Peralta (1996) señala que "La teoría del currículum entendida hoy en día como una disciplina que 
pretende estudiar las relaciones entre la teoría y la práctica educacionales, y entre ésta y las sociedades y culturas con las cuales interactúa ..."(p.19) permite aclarar que el concepto curricular dependerá de las experiencias y de los fines que se quieran alcanzar. No obstante, se debe tomar en cuenta que las líneas de pensamiento curricular definen a los grupos sociales que intervienen el fenómeno educativo. En el caso de la Escuela de Enfermería, el constructivismo es la línea de pensamiento orientador en el plan de estudios de la Licenciatura en Enfermería de la Universidad de Costa Rica.

Por otro lado, Stenhouse, citado por Sacristán (1991), afirma que:

un currículo...si es valioso, a través de materiales y criterios para llevar a cabo la enseñanza, expresa toda una visión de lo que es el conocimiento y una concepción del proceso de la educación. Proporciona un marco donde el profesor (a) puede desarrollar nuevas habilidades, relacionándolas con las concepciones del conocimiento y del aprendizaje.(p.14).

No obstante, la investigadora considera que el aporte más elaborado es el presentado por Alicia de Alba (1994), al afirmar lo siguiente:

Noción de curriculum. Por curriculum se entiende a la síntesis de elementos culturales (conocimientos, valores, costumbres, creencias, hábitos) que conforman una propuesta políticoeducativa pensada e impulsada por diversos grupos y sectores sociales, cuyos intereses son diversos y contradictorios que algunos tiendan a ser dominantes o hegemónicos, y otros tiendan a oponerse y resistirse a tal dominación o hegemonía. Síntesis a la cual se arriba a través de diversos mecanismos de negociación e imposición social. Propuesta conformada por aspectos estructurales-formales y procesales-prácticos, así como por dimensiones generales y particulares que interactúan en el devenir de las curricula en las instituciones sociales educativas. Devenir curricular cuyo carácter es fundamentalmente histórico y no mecánico y lineal. Estructura y devenir que se conforman y expresan a través de distintos niveles de significación. (pp. 38-39)

Lo citado hace alusión no solo a los elementos que deben estar inmersos dentro de una propuesta curricular, sino también a la pertinencia sociocultural y a la propia estructura histórica. Además, la investigadora añadiría flexibilidad, donde exista la posibilidad de modificar y cambiar aquellos aspectos que los requiere el contexto en donde se desarrolle. Es por lo anterior, que las instituciones educativas son transmisoras sociales de cultura, de formas de pensamiento y de formación de profesionales; tal es el caso de la Escuela de Enfermería, encargada y responsable de la formación de enfermeros y enfermeras en Costa Rica.

La responsabilidad y compromiso social lleva a la institución a realizar investigación educativa (Vitale, s.f.) que le brinde la información necesaria para gestar nuevas propuestas o mejorar aquellas que están en acción. En este caso particular, toda la Escuela de Enfermería gira entorno al currículum y, por ende, a todos los aspectos o elementos contenidos en él. De esta manera, el currículum posee una trascendencia histórica, social, cultural, política e ideológica. 
Por lo anterior, en un estudio curricular se deben identificar tres manifestaciones sobre el proceso educativo, no desvinculadas entre sí ni en el orden en que se exponen. Galán y Marín (s.f.) hacen referencia a esto y mencionan:

...currículo formal, es decir, la determinación y organización explícita de los contenidos, objetivos, etc., en el llamado plan de estudios, dirigido a una práctica profesional en formación, y a su justificación social. Una segunda, es la relativa al currículo vivido o real, esto es, a la puesta en marcha en la cotidianidad educativa de este plan de estudios, con todas las instancias que intervienen, como son la administrativa, la docente, la estoclar, la institucional, etc. Y la última forma está referida al currículo oculto, o sea a los elementos ideológicos implícitos en cualquier currículo. (pp. 27-28)

En el currículum, se deben diferenciar dos aspectos importantes: el desarrollo curricular, encaminado al conjunto de procesos que definen la formación profesional específica, y el diseño curricular, esto es, todos los elementos de un programa académico que determinan la organización de la enseñanza.

Respecto de este artículo, que nace de una investigación educativa (Restrepo, 1996) sobre el análisis curricular de la propuesta educativa de Enfermería de la Escuela de Enfermería de la Universidad de Costa Rica, tiene por objetivo presentar los resultados que corresponden a la pertinencia del plan de estudios en el contexto sociolaboral.

Es importante destacar que la pertinencia es un factor que considera que los graduados deben ser profesionales actualizados y capaces de desarrollar destrezas según se le solicita en el campo laboral y no únicamente personas capaces de buscar un empleo y adaptarse a él. Esto porque los empleadores solicitan profesionales creativos dado el cambio continúo que se suscita en los diversos empleos. De acuerdo a Corzo y Marcano (2009):

...valorar la pertinencia no será tarea fácil, especialmente si se adopta el concepto amplio de pertinencia social, ya que, exige examinarla no sólo en cuanto a su trabajo académico, sino también en función de los objetivos y de la misión que la educación debe cumplir en la sociedad. (p.151)

Corzo y Marcano (2009) señalan que "la pertinencia social denota las respuestas que proporciona el currículo a las necesidades del entorno y el mundo del trabajo" (p.152).

Como se explicitó, el plan de estudios no es una estructura fija, sino dinámica, la cual, además, refleja las metas y experiencias educativas a ser alcanzadas y proporcionadas. También, este debe ser pertinente con las diferentes estructuras socioculturales (Pérez, 2006). El plan de estudios cambia con el tiempo, por lo cual es importante el análisis y la reflexión continua, pues esta permite llevar a cabo las mejoras y los ajustes necesarios que respondan a las necesidades sociales del entorno laboral para el cual se forma el estudiantado. 


\section{MATERIALES Y MÉTODOS}

Este estudio se enmarcó dentro de los postulados que presenta el enfoque cuantitativo de tipo descriptivo (Hernández, 1997; Cea D’Ancona, s.f.). La población estuvo constituida por 74 egresados de instituciones públicas y privadas de la generación 2008 y por los entes empleadores en donde laboraban estos. Se aplicó un muestreo probabilístico simple, incrementando el intervalo de confianza a 10, con el fin de no afectar el nivel de confianza (95\%). Lo anterior se realiza para poblaciones pequeñas; la muestra, entonces, quedó en 43 egresados, pero esto no afecta el poder del tamaño de la muestra ni el nivel de confianza. En el caso de entes empleadores, se eligió seis instituciones en donde laboraban los sujetos seleccionados en la muestra. Estas fueron: EBAIS de Tres Ríos, Hospital México, Hospital La Católica, Hospital Rafael Angel Calderón Guardia, Hospital San Vicente de Paul, Hospital Max Peralta de Cartago.

Se analizaron tres dimensiones: formación del egresado, desempeño en campos no tradicionales y trascendencia de la acción laboral en la atención a la persona. Las variables correspondientes a cada una de esas dimensiones fueron: las prácticas profesionales, el contexto educativo, la metodología pedagógica, el desarrollo de habilidades individuales e interpersonales para el desempeño de su ejercicio laboral, las áreas del desempeño, los criterios de evaluación de los empleadores y la interacción con las y los usuarios.

En cuanto a la recolección de los datos, se aplicó un cuestionario estructurado, sin intermediarios, a los individuos; las respuestas las marcan los y las participantes (Hernández, Fernández y Baptista, 2010). Este instrumento exploró la percepción que manejan acerca de su formación en relación con las prácticas dominantes y emergentes, los escenarios y experiencias de aprendizaje y la incorporación de los ejes transversales del plan de estudios: liderazgo, género y derechos humanos. Además, se buscó conocer las áreas de desempeño profesional, las condiciones laborales y el grado de satisfacción de la formación académica. También se aplicó una entrevista semiestructurada a los entes empleadores de instituciones públicas y privadas. Su objetivo consistió en determinar el desempeño de las y los egresados en campos no tradicionales y los criterios y tipos de evaluación que implementan.

Para el procesamiento del cuestionario, se utilizó el programa StatisticPackage Social Science (SPSS); posteriormente se elaboraron tablas y gráficos. La entrevista semiestructurada permitió fortalecer las respuestas de los y las egresadas.

\section{Consideraciones éticas}

Esta investigación fue desarrollada bajo diversos principios éticos. El principio de autonomía se respetó al aclararle a cada persona sus posibilidades de aceptar o negar su participación voluntaria en el estudio o la posibilidad de solicitar, en cualquier momento, ser excluido de la investigación. Además, se garantiza la beneficencia y no maleficencia, desde el objetivo general de la investigación, pues al analizar la pertinencia en el contexto socio laboral, la unidad académica podrá tomar acciones de mejoramiento, en busca de la excelencia en la formación de profesionales. También, a cada informante se le explicó la naturaleza y la finalidad de la investigación, a la vez que se mantuvo la privacidad y el anonimato en todo momento. 


\section{RESULTADOS} anterior.

A continuación, se presentan los resultados según las dimensiones y variables anotadas en el apartado

\section{Formación del egresado}

Para esta dimensión, se generaron cuatro variables: prácticas profesionales, el contexto educativo, la metodología pedagógica y el desarrollo de habilidades personales e interpersonales para el ejercicio de la práctica profesional. Con respecto a la primera variable, fue relevante el tipo de prácticas (dominantes y emergentes). Al respecto, obsérvese la tabla 1 , en donde se evidencia según la opinión de los egresados, las prácticas dominantes e importantes para su desarrollo profesional y aquellas prácticas emergentes que han tenido que realizar según las exigencias en su campo laboral.

\section{Tabla 1}

Escuela de Enfermería, UCR: Distribución de las respuestas de los egresados según tipo de prácticas dominantes y emergentes que realizan y han realizado en su

desempeño profesional. 2011

(Frecuencias absolutas)

\begin{tabular}{l|c|c|c|c}
\hline \multicolumn{1}{c|}{ Tipo de práctica } & \multicolumn{2}{c|}{ Dominante } & \multicolumn{2}{c}{ Emergente } \\
\hline & Realiza & Ha realizado & Realiza & Ha realizado \\
\hline Cuidado directo & 42 & 24 & - & - \\
\hline Educación & 11 & 5 & - & - \\
\hline Supervisión y gerencia & 24 & 16 & - & - \\
\hline Elaboración y revisión de Protocolos & - & 2 & - & 2 \\
\hline Incursión en medicina alternativa & - & - & - & 1 \\
\hline Manejo de tecnologías médicas & - & - & 7 & - \\
\hline Investigación & - & 4 & & \\
\hline
\end{tabular}

Otro aspecto importante de analizar en la investigación fue la percepción que tenían los egresados sobre los saberes que posee como parte de su formación académica. (Obsérvese la tabla 2)

\section{Tabla 2}

Escuela de Enfermería, UCR: Distribución de la opinión de egresados según su percepción sobre el conocimiento adquirido en la formación. 2011

(Frecuencias absolutas)

\begin{tabular}{l|c}
\hline \multicolumn{1}{c|}{ Percepción } & $\begin{array}{c}\text { No. } \\
\text { respuestas }\end{array}$ \\
\hline Buen conocimiento teórico y poca práctica & 23 \\
\hline Adecuado conocimiento & 8 \\
\hline Amplio conocimiento teórico y práctico & 6 \\
\hline Débil conocimiento en fisiopatología e intervención tercer nivel & 4 \\
\hline Insuficiente conocimiento para desempeñar puestos de jefatura & 2 \\
\hline \multicolumn{1}{c}{ Total } & $\mathbf{4 3}$ \\
\hline
\end{tabular}




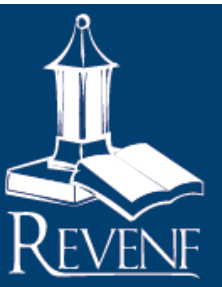

Una cantidad importante de opiniones (23) señala que el plan de estudios les brinda un buen conocimiento para desempeñarse en el campo laboral, sin embargo de igual forma perciben que la práctica resulta poca para abordar todas las exigencias del medio laboral actual. Asimismo, se debe destacar que son mayores las opiniones positivas al conocimiento recibido que aquellas que denotan deficiencias en la formación.

Otra variable que se consideró interesante para el análisis de este estudio fue la opinión que tienen los egresados sobre el contexto educativo. Para esto se investigó acerca de los escenarios de aprendizaje y el tipo de experiencias de aprendizaje desarrolladas en los diferentes módulos que conforman el plan de estudios y en el último curso "Gestión del Cuidado". Los datos se muestran en la tabla 3.

\section{Tabla 3}

\section{Escuela de Enfermería, UCR: Distribución de la opinión de egresados según escenarios de aprendizaje en módulos y el curso de Práctica de Enfermería. 2011}

\begin{tabular}{|c|c|c|c|c|c|c|c|c|c|c|c|c|c|c|c|c|c|c|c|c|c|}
\hline \multirow[t]{3}{*}{ Opinión } & \multirow{2}{*}{\multicolumn{3}{|c|}{ Niñez sana }} & \multirow{2}{*}{\multicolumn{3}{|c|}{ Niñez mórbida }} & \multicolumn{3}{|c|}{ Adolescente } & \multicolumn{3}{|c|}{ Adultez sana } & \multicolumn{3}{|c|}{$\begin{array}{l}\text { Adultez } \\
\text { mórbida }\end{array}$} & \multicolumn{3}{|c|}{ Adultez mayor } & \multicolumn{3}{|c|}{ Gestión } \\
\hline & & & & & & & & No. & & & $\mathbf{N}$ & & & No. & & & No & & & No & \\
\hline & Si & $\begin{array}{l}\mathbf{N} \\
\mathbf{0}\end{array}$ & $\mathbf{T}$ & $\mathbf{S i}$ & $\begin{array}{c}\mathbf{N} \\
\mathbf{0}\end{array}$ & $\mathbf{T}$ & $\mathbf{S i}$ & $\begin{array}{l}\mathbf{N} \\
\mathbf{0}\end{array}$ & $\mathbf{T}$ & $\mathbf{S i}$ & I & 7 & $\mathbf{S i}$ & $\begin{array}{l}\mathbf{N} \\
\mathbf{0}\end{array}$ & $\mathbf{T}$ & $\mathbf{S i}$ & $\begin{array}{l}\mathbf{N} \\
\mathbf{0}\end{array}$ & $\mathbf{T}$ & $\mathbf{S i}$ & $\begin{array}{l}\mathbf{N} \\
\mathbf{0}\end{array}$ & $\mathbf{T}$ \\
\hline $\begin{array}{l}\text { Idóneo para } \\
\text { el logro de } \\
\text { objetivos }\end{array}$ & 30 & 13 & $\begin{array}{l}4 \\
3\end{array}$ & 23 & 20 & $\begin{array}{l}4 \\
3\end{array}$ & 29 & 14 & $\begin{array}{l}4 \\
3\end{array}$ & 25 & 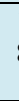 & & 24 & 19 & $\begin{array}{l}4 \\
3\end{array}$ & 25 & $\begin{array}{l}1 \\
8\end{array}$ & $\begin{array}{l}4 \\
3\end{array}$ & 26 & 17 & 43 \\
\hline $\begin{array}{l}\text { Brindó } \\
\text { suficientes } \\
\text { experiencias } \\
\text { clínicas }\end{array}$ & 25 & 18 & $\begin{array}{l}4 \\
3\end{array}$ & 23 & 20 & $\begin{array}{l}4 \\
3\end{array}$ & 18 & 25 & $\begin{array}{l}4 \\
3\end{array}$ & 16 & 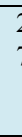 & & 23 & 20 & $\begin{array}{l}4 \\
3\end{array}$ & 25 & $\begin{array}{l}1 \\
8\end{array}$ & $\begin{array}{l}4 \\
3\end{array}$ & 17 & 26 & 43 \\
\hline $\begin{array}{l}\text { Fue } \\
\text { suficiente el } \\
\text { tiempo } \\
\text { asignado } \\
\end{array}$ & 26 & 17 & $\begin{array}{l}4 \\
3\end{array}$ & 19 & 24 & $\begin{array}{l}4 \\
3\end{array}$ & 23 & 20 & $\begin{array}{l}4 \\
3\end{array}$ & 20 & 3 & & 17 & 26 & $\begin{array}{l}4 \\
3\end{array}$ & 19 & $\begin{array}{l}2 \\
4\end{array}$ & $\begin{array}{l}4 \\
3\end{array}$ & 15 & 28 & 43 \\
\hline $\begin{array}{l}\text { Se tuvo que } \\
\text { modificar la } \\
\text { práctica }\end{array}$ & 1 & 42 & $\begin{array}{l}4 \\
3\end{array}$ & 4 & 39 & $\begin{array}{l}4 \\
3\end{array}$ & 1 & 42 & $\begin{array}{l}4 \\
3\end{array}$ & 5 & 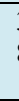 & & 8 & 35 & $\begin{array}{l}4 \\
3\end{array}$ & 4 & $\begin{array}{l}3 \\
9\end{array}$ & $\begin{array}{l}4 \\
3\end{array}$ & 5 & 38 & 43 \\
\hline $\begin{array}{l}\text { No respondió } \\
\text { a los } \\
\text { objetivos } \\
\text { planteados }\end{array}$ & 0 & 43 & $\begin{array}{l}4 \\
3\end{array}$ & 4 & 39 & $\begin{array}{l}4 \\
3\end{array}$ & 4 & 39 & $\begin{array}{l}4 \\
3\end{array}$ & 3 & . & & 4 & 39 & $\begin{array}{l}4 \\
3\end{array}$ & 3 & $\begin{array}{l}4 \\
0\end{array}$ & $\begin{array}{l}4 \\
3\end{array}$ & 5 & 38 & 43 \\
\hline
\end{tabular}

Idoneidad $^{1}$ para el logro de los objetivos: más de la mitad de los y las egresados opinaron que los escenarios de aprendizaje de los diferentes módulos son idóneos para el logro de los objetivos.

Los escenarios que brindaron suficientes experiencias clínicas son los módulos de la niñez y la adultez mayor. Debe destacarse que el módulo que brindó menor experiencia fue la adultez sana seguida por el curso de Gestión del cuidado.

Además, se les pidió a los y las egresadas que emitieran su opinión con respecto al tipo de experiencias de aprendizaje que fueron significativas para su formación. Mencionaron que tanto las actividades de aprendizaje

\footnotetext{
${ }^{1}$ Idoneidad: según Calmels, D. (2008) "Joan Corominas (1973) define el concepto de "idóneo", derivado del latín idoněus, como «adecuado, apropiado» (María Moliner agrega a la definición «apto»). Podríamos establecer una diferencia entre el concepto de idóneo como capacidad y condición de posibilidad de ejercicio de una acción determinada, usado como adjetivo, y el término Idóneo con mayúscula, designando la figura de un profesional que se desempeña en una práctica educativa o terapéutica sin contar con el título correspondiente y habilitante. En este caso hablamos de una persona Idónea en psicología, psicomotricidad, etc."'La persona Idónea se caracteriza por contar a su favor con una experiencia suficiente y constante en una práctica determinada. Si bien el Idóneo debe contar con una formación teórica, lo que caracteriza su reconocimiento es su práctica clínica, educativa, terapéutica, habilitante para el ejercicio de un rol determinado. Sin embargo, para su inserción profesional en algunos organismos oficiales el Idóneo deberá someterse a una «prueba de idoneidad»" (http://www.aapsicomotricidad.com.ar/idoneo.pdf)
} 
desarrolladas propiamente en la práctica de enfermería (74 respuestas positivas) así como la investigación (17 respuestas) las experiencias que fortalecen el sustento teórico (15 respuestas) y las experiencias de educación para la salud (13 respuestas) fueron las actividades que tuvieron mayor peso en su formación.

Un número significativo de los sujetos encuestados (17) menciona el haber participado en actividades extracurriculares. Entre ellas, las más nombradas fueron las actividades educativas, seguidas por las de investigación. No obstante, cabe destacar que más de la mitad (26) refiere no haber participado en ninguna de ellas.

Con respecto al desarrollo de su práctica profesional, se les solicitó a los egresados participantes en el estudio que emitieran su opinión sobre la incorporación en su práctica clínica de liderazgo, derechos humanos y género. Véase los datos en la tabla 4.

\section{Tabla 4}

\section{Escuela de Enfermería: Distribución de la opinión de egresados según la incorporación en su ejercicio} profesional el liderazgo, derechos humanos y género. 2011

(Frecuencias absolutas)

\begin{tabular}{l|c|l|l|l|c}
\hline \multicolumn{1}{c|}{ Liderazgo } & $\begin{array}{l}\text { No. } \\
\text { Resp. }\end{array}$ & Derechos Humanos & $\begin{array}{l}\text { No. } \\
\text { Resp }\end{array}$ & \multicolumn{1}{|c|}{ Género } & $\begin{array}{c}\text { No. } \\
\text { Resp }\end{array}$ \\
\hline Trabajo en equipo & 10 & Equidad e igualdad en el trato & 11 & $\begin{array}{l}\text { Igualdad, } \\
\text { equidad y } \\
\text { respeto }\end{array}$ & 22 \\
\hline Coordinaciones & 7 & $\begin{array}{l}\text { Contempla los derechos } \\
\text { humanos en el trato con las } \\
\text { personas }\end{array}$ & 22 & $\begin{array}{l}\text { Uso de lenguaje } \\
\text { inclusivo }\end{array}$ & 2 \\
\hline $\begin{array}{l}\text { Conocimiento teórico y } \\
\text { práctico }\end{array}$ & 3 & & & $\begin{array}{l}\text { No responde, no } \\
\text { me interesa }\end{array}$ & 6 \\
\hline Comunicación & 4 & & & & \\
\hline $\begin{array}{l}\text { Negociación y actitudes } \\
\text { positivas }\end{array}$ & 1 & & & & \\
\hline $\begin{array}{l}\text { Formulación y ejecución } \\
\text { de proyectos }\end{array}$ & 3 & & & & \\
\hline Toma de decisiones & 1 & & & & \\
\hline Motivación & 3 & & & \\
\hline
\end{tabular}

\section{Desempeño en el campo laboral}

En esta dimensión, se consideraron las áreas de desempeño profesional. Dentro de esta variable, interesó conocer sobre los siguientes aspectos: los tipos de campos de trabajo -tanto tradicionales como no tradicionales-, la condición laboral, el grado de satisfacción de su formación académica para responder a la demanda laboral y las clases de formación requerida para complementar su práctica laboral.

En relación al desempeño laboral de las y los egresados, la mayoría (39) trabaja en el área hospitalaria, sin embargo, puede apreciarse en la tabla 5, que todos los campos donde ejercen han sido espacios áulicos de enseñanza a través de todo el plan de estudios de la carrera de Enfermería. Esto podría suponer que la formación en dichos escenarios les ha brindado las herramientas para desarrollarse, posteriormente, en su vida laboral. 
En Costa Rica, el sector hospitalario es bastante amplio; por esto, gran parte de los egresados son acogidos por este empleador. En resumen, resulta posible determinar que existe coherencia entre la formación y los campos de desempeño laboral de las y los enfermeros egresados.

Tabla 5

Escuela de Enfermería, UCR: Distribución de respuestas de los egresados sobre los campos de desempeño laboral. 2011

(Frecuencias absolutas)

\begin{tabular}{l|c}
\hline \multicolumn{1}{c|}{ Campos de desempeño } & $\begin{array}{c}\text { No. } \\
\text { Respuestas }\end{array}$ \\
\hline $\begin{array}{l}\text { Cuidado de la persona adulta } \\
\text { mayor }\end{array}$ & 3 \\
\hline Enfermería de empresa & 5 \\
\hline Instituciones educativas & 1 \\
\hline Adolescentes y adultos jóvenes & 1 \\
\hline Hospital & 39 \\
\hline Primer nivel de atención & 7 \\
\hline Segundo nivel de atención & 1 \\
\hline Privado & 5 \\
\hline Auxiliar & 4 \\
\hline Administrativo & 3 \\
\hline Investigación & 5 \\
\hline Cuidado a domicilio & 2 \\
\hline Docente & 6 \\
\hline Consultorías & 2 \\
\hline Empresario & 2 \\
\hline Vendedor & 1 \\
\hline Atención pre hospitalaria & 1 \\
\hline
\end{tabular}

Los y las egresadas manifiestan, en un alto número (35), sentirse satisfechos con la formación recibida (véase gráfico 1). Su respuesta es muy congruente con lo evidenciado en la tabla 5 sobre la competencia que posee el egresado de desarrollarse en diversos campos laborales, esto debido a la amplia formación que le brinda la Escuela de Enfermería. Algunas respuestas brindadas son las siguientes:

"Me realicé como el profesional que siempre quise ser"; "Muy buen plan de estudios"; "Al llegar a la práctica contaba con herramientas suficientes para innovar e incursionar". 
Las respuestas consideradas entre poco satisfecho e insatisfecho expresan que necesitan que se les brinde mayor oportunidad de realizar práctica en los campos clínicos asignados. Así se expresaron algunos (a): "Hace falta más destreza práctica", "Brinda las herramientas para llegar a ser un buen profesional aunque falta práctica".

Esto es consistente con lo encontrado en la tabla 3 sobre el tiempo asignado en los módulos así como en el curso de Gestión del Cuidado, que los sujetos encuestados lo calificaron de insuficiente.

\section{Trascendencia de la acción laboral en la atención a la persona}

Las variables consideradas al analizar la transcendencia de la acción laboral en la atención a la persona fueron los "criterios de evaluación que usan los empleadores" y la "interacción con la persona". De esta manera, se contempló el tipo de vínculo que establece el o la egresada con las personas y la posición epistemológica en relación con la trascendencia en el accionar laboral.

En cuanto a las variables anteriores, se pudo observar que casi la totalidad de la muestra (41) considera que el respeto es el valor que priva en las relaciones que establecen. También, manifestaron "la relación profesional" (13 respuestas) como otra forma de establecer nexos con las personas que atiende en su ambiente laboral y "la confianza" con 11 respuestas. Esto es parte de la formación en cuanto a la percepción de su identidad profesional, y el trato con la persona, el cual ha trascendido a una relación más horizontal. Esto concuerda con la autonomía de los pacientes y la formación del estudiantado en aspectos de Bioética, Ética y Derechos Humanos.

En términos generales, los empleadores entrevistados poseen una excelente opinión acerca del desempeño de las y los egresados de la Escuela de Enfermería. Algunos enfatizan en que se debe mejorar el aspecto de las funciones gerenciales; incluso refieren que algunos individuos se han visto obligados a complementar su conocimiento en relación con determinada temática, esto para poder llevar a cabo su trabajo. (Entrevistado TR). Lo anterior coincide con lo señalado por los y las egresadas al mencionar que han tenido que realizar estudios complementarios, entre ellos capacitaciones sobre conocimientos teóricos -con 48 respuestas- y sobre gerencia con 13 respuestas-, para desempeñarse a cabalidad en su trabajo.

Finalmente, se les solicitó a los participantes que emitieran su opinión con respecto a la relación entre la posición epistemológica en su formación y la trascendencia de esta en el accionar laboral. Se puede observar que la mayoría de las respuestas (14) se inclinan hacia "conocimiento profesional", "generar cambios positivos" (7) y "calidad del cuidado" (3). Sin embargo, las respuestas emitidas no brindan suficiente información para analizar la posición epistemológica que se plantea en el plan de estudios, la cual debe permear la formación del estudiantado y cual debería de evidenciarse en su práctica profesional.

\section{DISCUSIÓN}

Es importante enfatizar que los datos reflejan la realidad y pertinencia del plan de estudios con el objeto de la enfermería: el cuidado. El tipo de práctica destacada por los encuestados es la atención directa, lo cual no resulta extraño, ya que, al ser Enfermería una disciplina preponderantemente práctica, era probable que los datos 
dieran cuenta tanto de lo anterior, como de la distribución del plan en la malla curricular con respecto a la cantidad de horas que dedica el estudiantado a desarrollar sus habilidades y destrezas para poder dar una intervención de enfermería de calidad.

No obstante, se debe tomar en cuenta que todo lo que concierne a la salud es dinámico y se encuentra en constante evolución y cambio; esto se evidencia en las prácticas emergentes a las que hacen referencia las y los egresados en los datos recolectados. Entre ellas, destaca el manejo de las tecnologías médicas, para lo cual se requiere capacitación constante que posibilita dar respuesta a las prácticas emergentes que se suscitan en su campo laboral.

No menos importante es el tipo de práctica, que mencionan las y los entrevistados, relacionadas con la educación para la salud y la supervisión y gerencia de las actividades que se desarrollan en sus lugares de trabajo, lo cual es coherente con los propósitos de la formación del estudiantado inmerso en el plan de estudios.

Un plan de estudios se debe caracterizar por evidenciar un equilibrio entre sus distintos elementos desde la teoría y la práctica. Sin embargo, la investigación detectó que el de Enfermería desarrolla temáticas actualizadas y suficientes para que el estudiantado se desempeñe en su campo laboral; la práctica es percibida por ellos y ellas como insuficiente para abordar las exigencias del medio laboral actual.

Otro aspecto relevante para los resultados es el tiempo asignado para desarrollar la teoría y la práctica, pues las y los encuestados lo señalaron como insuficiente, enfatizando notablemente en el segundo aspecto. No obstante, esta última no fue modificada, ya que los objetivos siempre fueron alcanzados. Se podría decir, entonces, que lo anterior demuestra cierta inconsistencia en la percepción del estudiantado al respecto. Sin embargo, el éxito de la práctica puede deberse a otros factores educativos, tales como la del docente para el desarrollo de las actividades educativas, la motivación docente-estudiante y las experiencias y oportunidades que los diversos campos clínicos ofrecen a los grupos de estudiantes. Esto no sustituye el retomar lo anotado antes para el mejoramiento de las experiencias de aprendizaje de los módulos y el curso de Gestión del cuidado.

Las experiencias de aprendizaje ofrecen al estudiantado la oportunidad de desarrollar habilidades y destrezas en su formación; su principal característica es el hecho de que el alumnado, como protagonista del proceso, se enfrenta a una serie de experiencias que lo fortalecen en el área de resolución de problemas. Además, ayudan a profundizar y abordar de manera creativa el aprendizaje planificado en un momento y escenario concreto. En ese sentido, las experiencias más significativas, según las respuestas del egresado, son aquellas que se desarrollan a través de la práctica clínica, probablemente también debido a la continuidad y articulación de los procesos que se describen en los diferentes módulos, y el curso de Gestión en Enfermería. Ambos tienen una estructura ordenada y lógica en el quehacer educativo para la enseñanza de la Enfermería.

Otra experiencia que guarda significado con un número importante de respuestas positivas es el tema de la investigación. Se debe notar que esta es un eje transversal del plan de estudios y que el estudiantado se ve expuesto a que sus prácticas estén basadas en aspectos metodológicos de la investigación, además de concluir su formación con la defensa pública de un trabajo final de graduación. 
Es importante mencionar que la metodología pedagógica de un plan de estudios no estaría completa si no se incluyeran actividades extracurriculares que enriquecen el aprendizaje y la enseñanza de la Enfermería. Estas se realizan fuera del horario académico y su participación es voluntaria; además, están orientadas hacia la formación integral que amplíe el horizonte cultural del estudiantado.

Respecto de los ejes transversales en el contexto educativo -de acuerdo con el folleto realizado por la Escuela de Enfermería con guía de la Comisión de Reconocimientos, Credenciales y Curriculum- estos acercan a los y las estudiantes de Enfermería a una realidad social, lo cual da sentido al curriculum. Lo anterior se explica porque la transversalidad está determinada por situaciones problemáticas o socialmente relevantes. En el caso de la Enfermería, los ejes transversales propuestos son: Derechos Humanos y Género, Liderazgo y, finalmente, Investigación. Estos constituyen grandes líneas que cruzan todo el planteamiento curricular del plan de estudios de la Licenciatura en Enfermería. La decisión de incorporar esas temáticas se gestó a partir de un espacio de reflexión de la Unidad Académica, esto en el momento de reestructurar el actual plan de estudios.

Al implementar esos ejes transversales, se pretende que los y las estudiantes, al finalizar su proceso de formación, introyecten los principios éticos y actitudinales democráticos, respetuosos, solidarios, proactivos, de pensamiento crítico, creativo, innovadores y con conciencia social, entre otros. Estos caracterizan a todo profesional en Enfermería que se desempeñe con excelencia en los distintos espacios de trabajo y en su vida personal. Sin embargo, los resultados mostraron -según la opinión de los y las egresadas- poca o nula introyección de los ejes transversales sobre Derechos Humanos y Género, y Liderazgo; únicamente se denotó rasgos de cada uno de ellos. Dada la importancia para el desarrollo integral del estudiantado y su desempeño laboral como profesión de "servicio" es importante que se considere el análisis de este aspecto.

Otros elementos que influyen en el desempeño laboral son la satisfacción, las características de cada persona, sus habilidades, cualidades y las necesidades que se manifiestan en el lugar de trabajo. Respecto de esto, la investigación denotó que la formación que se le brinda al estudiantado, según los y las egresadas, les proporciona suficientes competencias para desarrollarse en una gran diversidad de campos. Al ser este una forma de proyección eficiente del empleado hacia las actividades o funciones que realiza para alcanzar los logros establecidos en la institución para la que trabaje. También, el respeto es considerado -desde la ética- como la esencia de las relaciones humanas de cualquier tipo, incluso el trabajo en equipo, es un elemento muy importante para el buen desempeño laboral.

De la misma forma que el desempeño laboral es un elemento muy importante para valorar el planeamiento curricular, lo es también la satisfacción que tienen las y los egresados con respecto a su formación académica. Lo provechoso en el aprendizaje está unido, indiscutiblemente, por la forma de aprender del estudiantado, de los modelos de aprendizaje que se le brindan en las diferentes situaciones que se le plantean a través de la carrera, siendo esto procesos dinámicos que pueden variar según el curso o el mismo docente que guía la construcción del saber del alumnado; por lo cual van a impactar de una u otra manera en la satisfacción de su formación.

Esta satisfacción, por ende, va a estar unida a la calidad de la educación que se imparte en un centro educativo, en este caso la Escuela de Enfermería, en donde influyen elementos que van desde el personal docente 
(en calidad, calidez y experticia, entre otros aspectos) hasta las instalaciones, la oferta educativa, los entornos virtuales, la comunicación y relaciones interpersonales, etc.

En el plan de estudios, se considera, a través de la carrera, el fomento del trabajo en equipo, coincidente con lo expresado por las y los egresadas en donde muchas de las actividades de aprendizaje de cursos y módulos tienen esta metodología didáctica y desde el eje transversal de Derechos Humanos y Género -que atraviesa toda la oferta educativa- se fortalecen los valores y entre ellos el respeto hacia las personas. Por tal motivo, no es de extrañar que los y las egresadas tengan introyectado ese valor y lo apliquen en su desempeño laboral.

En relación con el accionar del estudiantado en su transcendencia personal, es importante destacar lo mencionado en una entrevista a Estela Quintar de la Universidad de Lasalle, quien explica que todo el proceso de formación (experiencias y vivencias) de un estudiante, lo atraviesa y lo hace crecer como sujeto histórico que eligió ser enfermera o enfermero. Por lo tanto, el espacio pedagógico se convierte en una posibilidad de ser, de pensar, de ser autónomos y tener sentido de futuro, de contribuir al cambio de los propios estudiantes y del contexto que le rodea; por lo cual un plan de estudios debe tener los elementos curriculares necesarios trascendentes con espacios de formación que logre un cambio en los estudiantes, provocando el deseo de un saber crítico para que ellos y ellas propongan alternativas de transformación de la realidad conjuntamente con las personas que le rodean. De ahí que, según la información recopilada en esta investigación, el estudiantado menciona en primera instancia el "conocimiento profesional" el cual está constituido de saberes que lo inducen a la transformación de sí mismo y de las otras personas en su accionar laboral y que generan cambios positivos.

En términos generales, se puede decir que la propuesta educativa correspondiente a la carrera de Licenciatura en Enfermería de la Universidad de Costa Rica guarda una estrecha pertinencia con el contexto socio-laboral. No obstante, los procesos educativos deben estar en continua revisión, actualización y mejoramiento, de acuerdo con los planteamientos que surgen a través del tiempo, para que el estudiantado se asegure que su formación va a responder a las exigencias en salud, sociales y laborales.

\section{CONCLUSIONES}

-Se requiere de un mayor tiempo para el desarrollo de experiencias de aprendizaje en los módulos y el curso de Gestión del Cuidado, así como mejoras internas, en cada uno, de la suficiencia e idoneidad de tales experiencias. Además, es importante estar en constante mejoramiento de la oferta educativa en términos de la cantidad de horas de práctica destinadas en el plan de estudios y la calidad de las experiencias clínicas.

-No hay una introyección cabal de los ejes transversales de derechos humanos y género así como del de Liderazgo.

-Hay una fuerte coherencia entre la formación y los campos de desempeño laboral de los y las egresadas.

-La formación en la temática de las funciones gerenciales no brinda las suficientes herramientas a los egresados y egresadas, ya que han tenido que complementar ese saber con cursos adicionales para poder llevar a cabo su trabajo. 


\section{AGRADECIMIENTOS}

Se agradece profundamente el apoyo brindado para concluir con éxito esta investigación.

Magister Antonieta Calvo Rodríguez, Directora colaboradora hasta Julio 2010.

M.Sc. Ligia Murillo Castro, Directora colaboradora, Julio 2010 a la fecha.

Comisión de Reconocimiento, Credenciales y Curriculum, 2006 a Febrero 2012

Dra. Ligia Rojas Valenciano, Coordinadora

Magister Ana Guzmán Aguilar, Miembro

Magister Viriam Leiva Díaz, Miembro

M.Sc. Mabel Granados Hernández, Miembro

Magister Rebeca Gómez, Miembro

Magister Derby Muñoz, Miembro

Comisión de Reconocimiento, Credenciales y Curriculum, Febrero 2012 a la fecha

Magister Rebeca Gómez, Coordinadora

M.Sc. Mabel Granados Hernández, Miembro

Magister Laura López, Miembro

Magister Dennis Carrillo, Miembro

Dra. Alba Carranza, Miembro

Magister Elena Mora Escalante

\section{Otros colaboradores}

Docentes de la Escuela de Enfermería de la Universidad de Costa Rica.

Centro de Evaluación Académica de la Universidad de Costa Rica.

Enfermeras y Enfermeros que laboran en instituciones de salud privada y pública.

Estudiantes de Enfermería de la Universidad de Costa Rica de la promoción del 2011.

\section{REFERENCIAS}

Alba de, A. (1994). Evaluación curricular: conformación del campo. México D. F: UNAM.

Brenes, E. y Porras, M. (1995).Teoría de la Educación. San José, Costa Rica: EUNED.

Calmels, D. (2008).El profesional Idóneo y la idoneidad profesional. Borrador. Comunicación especialmente preparada para compartir con los colegas de la AAP. http://www.aapsicomotricidad.com.ar/idoneo.pdf

CeaD’Ancona, M.A.(s.f.). Metodología Cuantitativa. Estrategias y Técnicas de investigación social. Madrid, España: Síntesis Sociológica. 
Comisión de Reconocimiento, Credenciales y Curriculum(2006).Folleto de ejes transversales. Escuela de Enfermería. Universidad de Costa Rica.

Corzo, L. y Marcano, N. (2009). Pertinencia del currículo de las instituciones de educación superior: un estudio cualitativo desde la teoría fundamentada. Multiciencias, 9(2), 149-156.

Escuela de Enfermería. (2004). Malla curricular del plan de estudios.

FAO. (1999). Elaboración participativa de planes de estudios para la educación y capacitación agrícola. Departamento de Desarrollo Sostenible. Recuperado de: http://www.fao.org/docrep/009/w9693s/w9693s00.htm

Galán, I. y Marín, E. (s.f.). Marco teórico para el Estudio del Rendimiento Escolar y Evaluación del Currículo. Santiago: Departamento de Servicos Educativos del CISE

Hernández, R., Fernández, C. y Baptista, P.(1997). Metodología de la Investigación. México D. F.: McGraw Hill.

Hernández, R., Fernández, C. y Baptista, P. (2010). Metodología de la Investigación. México D. F.: McGraw Hill.

Peralta, V. (1996). Teoria del currículo. México D. F.: Ediciones Morata.

Pérez, T. (2006). La pertinencia de la Educación ¿pertinente con qué? Recuperado de http://www.plandecenal.edu.co/html/1726/articles-308333_archivo.pdf

Quintar, E. (s.f.). Entrevista sobre el proceso de formación. Universidad de Lasalle.

Restrepo, B. (1996). Investigación en Educación. Programa de Especialización en Teoría, Métodos y Técnicas de Investigación Social. Bogotá: Instituto Colombiano para el Fomento de la Educación Superior.

Rojas, L. (2004). Factores asociados a la repitencia de los y las estudiantes que cursan setimo año en colegios académicos, diurnos y públicos: un análisis de niveles múltiples. (Tesis doctoral). Universidad Estatal a Distancia, San José, Costa Rica.

Sacristán, J.(1991). Investigación y Desarrollo del Currículum. Madrid: Editorial Síntesis.

StatisticPackage Social Science. Manual para el uso del software SPSS.

Vitale, C. (s.f.). La Investigación Educativa. Recuperado de

http://www.fhumyar.unr.edu.ar/escuelas/3/materiales $\% 20 \mathrm{de} \% 20$ catedras/trabajo $\% 20 \mathrm{de} \% 20$ campo/adscripcion.ht $\underline{\mathrm{m}}$ 